\title{
BMJ Open A national survey exploring oral healthcare service provision across Australian community pharmacies
}

\author{
Christopher R Freeman, ${ }^{1}$ Nabilah Abdullah, ${ }^{1}$ Pauline $\mathrm{J} \mathrm{Ford,}{ }^{2}$ Meng-Wong Taing ${ }^{1}$
}

To cite: Freeman CR, Abdullah N, Ford PJ, et al. A national survey exploring oral healthcare service provision across Australian community pharmacies. BMJ Open 2017;7:e017940. doi:10.1136/ bmjopen-2017-017940

- Prepublication history and additional material for this paper are available online. To view these files please visit the journal (http://dx.doi.org/10. 1136/bmjopen-2016-017940).

Received 28 May 2017 Revised 6 July 2017 Accepted 8 August 2017

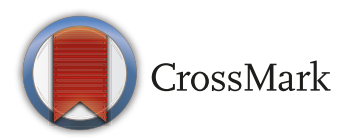

${ }^{1}$ School of Pharmacy, The University of Queensland, Brisbane, Queensland, Australia

${ }^{2}$ School of Dentistry, The University of Queensland, Brisbane, Queensland, Australia

Correspondence to Dr Meng-Wong Taing; m.taing@uq.edu.au

\begin{abstract}
Objectives This study investigated pharmacists' and pharmacy assistants' current practices and perspectives with regard to oral healthcare provision across Australian community pharmacies.
\end{abstract}

Design Cross-sectional study. A questionnaire for each pharmacist and pharmacy assistant cohort was developed and administered by online or postal means. Pearson's $\chi^{2}$ test was used to examine relationships between categorical variables.

Participants Pharmacists and pharmacy assistants working within 2100 randomly selected Australian community pharmacies.

Results The overall response rate was 58.5\% (644/1100) for the pharmacist cohort and $28 \%$ (280/1000) for the pharmacy assistant cohort. This represents pharmacy staff responses from 803 community pharmacies across Australia (approximately 14.6\%, 803/5500 of community pharmacies nationally). Overall, the majority of pharmacists $(80.2 \% ; 516 / 644)$ and pharmacy assistants (83.6\%; 234/280) reported providing oral health advice/ consultations to health consumers up to five times each week. More than half of community pharmacists and pharmacy assistants were involved in identifying signs and symptoms for oral health problems; and the majority believed health consumers were receptive to receiving oral health advice. Additionally, more than $80 \%$ of pharmacists and $60 \%$ of pharmacy assistants viewed extended oral healthcare roles positively and supported integrating them within their workplace; extended roles include provision of prevention, early intervention and referral to oral healthcare services. The most commonly reported barriers to enhance pharmacy staff involvement in oral healthcare within Australian community pharmacies include lack of knowledge, ongoing training and resources to assist practice.

Conclusion This study highlights that Australian pharmacists have an important role in oral health and provides evidence supporting the need for growing partnerships/collaborations between pharmacy and dental healthcare professionals and organisations to develop, implement and evaluate evidence-based resources, interventions and services to deliver improved and responsive oral healthcare within Australian communities.

\section{INTRODUCTION}

Good oral health is essential for quality of life and for general health. ${ }^{1}$ It can be defined

\section{Strengths and limitations of this study}

This study describes oral health services provided by Australian pharmacy staff and explores attitudes towards implementation of prevention, early intervention and referral oral health services in community pharmacies.

- The sample size for this study included data from 644 pharmacists and 280 pharmacy assistants which captures a range of Australian pharmacist and pharmacy assistant responses. This is the most comprehensive study known assessing Australian pharmacists' and pharmacy assistants' current practices and perspectives with regard to oral healthcare provision across Australian community pharmacies.

- Responses were self-reported and may be subject to respondent recall and social desirability bias. Participantresponses relating to weekly consultations and oral health product recommendations was not contextualised with respect to pharmacy turnover/ sales/size of pharmacy data.

as being free from any disease or disorder limiting an individual's ability and capacity in biting, chewing, speaking, smiling and psychosocial well-being. ${ }^{1}$ Oral disease is a considerable burden to the community with oral healthcare costs ranking as the second most expensive disease group in Australia (with cardiovascular disease the highest ranking). ${ }^{23}$ Almost all Australians experience an oral or dental problem in their lives, and expenditure in Australia on dental health services (excluding hospitals) was \$A8.7billion in 2012-2013, equivalent to approximately $6.5 \%$ of total national health expenditure. ${ }^{2}$ Poor oral health is associated with complex interactions between individual, structural, social and economic factors. These include geographic, cultural and financial access issues and individual health behaviours. ${ }^{4}$

In the majority of cases, the high cost of oral healthcare can be avoided by effective prevention strategies. ${ }^{15}$ In Australia, potentially preventable oral conditions include 
tooth decay, gum disease and oral cancer. ${ }^{6}$ Disadvantaged members of the community including low-income earners, the elderly and remote/rural and regional residents are less likely to receive preventive care services and are at higher risk of poor oral health compared with the general population. ${ }^{4}$ The WHO, International Association for Dental Research and World Dental Federation have established Global Oral Health Goals intended to provide a framework and guide health policy-makers at the national, regional and local levels regarding standards for oral health. Two objectives from this proposal advocate integration of non-dental healthcare providers for oral health promotion and surveillance of oral diseases and disorders. ${ }^{7}$ Numerous initiatives have been developed to support non-dental practitioner involvement in oral healthcare promotion, many of which centre around provision of prevention, early intervention and referral to oral healthcare services. ${ }^{8-11}$ There are limited studies reporting community pharmacy involvement or participation in the provision of any prevention, early intervention and referral oral health services.

In Australia, there are approximately 5500 community pharmacies well distributed and accessible in metropolitan, suburban, rural and remote regions. ${ }^{12}{ }^{13}$ On average, each person in Australia visits a community pharmacy up to 14 times per year. ${ }^{1415}$ Community pharmacy staff are trustworthy, knowledgeable and well-respected healthcare professionals that play a critical role in delivering quality healthcare to people of all income levels and stages of life. ${ }^{16}$ Services are generally provided without charge or need for an appointment and are usually in close proximity to people's homes. Pharmacists and pharmacy assistants are often the first or only point of contact with a health professional particularly for disadvantaged population groups including the elderly, who frequently visit their pharmacy for general and medication-related health advice. ${ }^{17-19}$ Patients with chronic conditions (eg, cardiovascular disease and diabetes) are also in regular contact with pharmacy staff for their prescriptions which presents an excellent opportunity to promote health advice through the common risk factor approach. ${ }^{20}{ }^{21}$ Because community pharmacy staff are readily accessible in the community ${ }^{12}$ and have established relationships with regular clients, they are ideally positioned to recognise and appropriately manage or refer a range of common oral health conditions for many population groups living in Australia. Community pharmacies may also logistically serve as ideal healthcare destinations to implement and deliver prevention, early intervention and referral oral health services to reduce the incidence of potentially preventable oral conditions including tooth decay, gum disease and oral cancer. Recent publications by the US Institute of Medicine and the Australian Dental Association (ADA) advocate for closer working relationships between non-dental healthcare professionals and the dental profession to build professional capabilities and undertake oral disease prevention, health promotion and screening to reduce oral health disparities within communities. ${ }^{22}{ }^{23}$ In the last 20 years, the traditional role of pharmacists (ie, dispensing and compounding medicines) has evolved to include a broader range of functions within primary healthcare. ${ }^{24-26}$ International studies and reports recognise the potential to enhance the role of pharmacy staff in oral health, given they are regularly consulted for a variety of oral health-related issues. ${ }^{19} 27-29$

Australian and international studies report pharmacy staff are eager to expand and undertake new roles in public health, expressed a strong interest in providing oral healthcare services to the community and viewed this role positively. ${ }^{29-33}$ Our previous study reported that community pharmacists valued their role as oral health advisors and that both pharmacists and pharmacy assistants were involved in handling the majority of oral health-related presentations in Australian community pharmacies. ${ }^{29}$ Nearly all pharmacists $(97 \%)$ also desired further education and training to benefit their practice in oral healthcare. The research is novel in that it is the first national study to use rigorous sampling methodology to evaluate population response estimates for both Australian pharmacists and pharmacy assistants with regard to oral healthcare service provision in community pharmacies. The study additionally explored staff views regarding implementation of prevention, early intervention and referral services which has not been previously evaluated. The aim of this study was to extend the findings of our previous work to gain further insight and knowledge about oral healthcare service provision across Australian pharmacies. The specific objectives of this study were to:

1. Identify services provided by Australian community pharmacy staff (pharmacists and pharmacy assistants) in oral healthcare and describe the range of over-thecounter (OTC) products stocked within Australian community pharmacies;

2. Evaluate Australian pharmacy staff attitudes and reported barriers towards provision of oral healthcare services and prevention, early intervention and referral to oral health services in Australian community pharmacies;

3. Determine pharmacy staff topics for future training, further support required and preferences for training;

4. Assess associations between staff characteristics and workplace/practice location variables with respect to oral health services/products provided, attitudes towards oral healthcare/preventive services and staff training preferences.

\section{METHODS}

Approval for the study was obtained by the University of Queensland's Human Research Ethics Committee in June 2016 (approval no 2016000067).

\section{Development of pharmacy staff questionnaires}

The questionnaires used in this study were based on the authors' (MWT, CF, PF) discipline-specific knowledge 
and experience in oral healthcare; and national Australian questionnaires created by Hussainy et al, however, reflecting topics relating to provision of oral healthcare and promotion services. Two questionnaires, one for pharmacy assistants and one for pharmacists were developed and comprised four sections to address the aims of this study. Section A comprised items relating to participant demographics; section B asked questions relating to oral health services provided at the pharmacy; section $\mathrm{C}$ asked questions relating to staff attitudes for provision of oral healthcare and preventive services in pharmacy and section D enquired about staff regarding training areas/ further support required. Apart from items relating to demographic information of participants, the content for both questionnaires were identical. The questionnaires were piloted for content and face validity by pharmacy and dentistry university academics $(\mathrm{n}=2)$, pharmacists $(n=5)$ and pharmacy assistants $(n=5)$. Changes regarding wording and formatting of questions were made and repiloted with pharmacists $(n=3)$ and pharmacy assistants $(n=3)$. Suggested changes were incorporated into the final questionnaire prior to national dissemination (see online supplementary 1 for the questionnaires).

\section{Sample size calculation and randomisation}

From approximately 5500 Australian community pharmacies, for each pharmacist and pharmacy assistant questionnaire, a sample size of 360 pharmacies for each cohort would provide a target $5 \%$ margin of error for population percentage estimates with a level of $95 \%$ confidence. A minimum $40 \%$ response rate was assumed (based on a previously used recruitment strategy by the authors ${ }^{34}$ ), therefore a randomised sample of 1000 Australian community pharmacies for each pharmacist and assistant cohort was selected (2000 pharmacies in total). An Australian pharmacy premises list obtained from pharmacy premises authorities from each Australian state/territory was used to randomly select a proportional number of pharmacies (20\%) from each state/territory. Randomisation was performed using Excel 2013.

\section{Participant recruitment}

All 2100 pharmacies were telephoned and pharmacists and pharmacy assistants from each cohort $(1100+1000$ pharmacies each) were invited to complete the questionnaire by online or postal means. Note, during the data collection phase, the pharmacist cohort included an additional 100 randomised pharmacies due to a distribution error in the online survey link. The researchers asked to speak with either the pharmacist or to the pharmacy assistant (that deals with OTC products) from the respective randomised list of pharmacies; if the participant agreed to participate in the research, they provided an email or postal address in which they received an online link to the survey or a hard copy version of the questionnaire. If they did not agree, they were counted as non-responders. This recruitment methodology has achieved higher response rates compared with recruitment through dissemination by pharmacy organisations (eg, Pharmaceutical Society of Australia (PSA)) previously reported. ${ }^{29}{ }^{34}$ Three follow-up reminders were made to participants via phone/email at twoweekly intervals. Data collection occurred between June and September 2016. All participants received a \$A10 retail gift card on completing the questionnaire to improve response rates.

\section{Data analysis}

The questionnaire responses were descriptively analysed using Statistical Package for Social Sciences (SPSS V.24) and Microsoft Excel 2013. Pearson's $\chi^{2}$ test was used to determine significant relationships between categorical variables; the significance level was set at $\mathrm{p}<0.05$. When contingency tables were larger than $2 \times 2$, adjusted standardised residuals was used to show cells which had larger or smaller counts than expected if two variables were considered independent. Missing data were excluded during analysis. ${ }^{35}$

\section{RESULTS}

The overall response rate was $58.5 \%(644 / 1100)$ for the pharmacist cohort and 28\% (280/1000) for the pharmacy assistant cohort. This represents pharmacy staff responses from 803 community pharmacies across Australia. The sample size for pharmacist and pharmacy assistant cohorts in this study resulted in a $3.6 \%$ and $5.7 \%$ margin of error for population percentage estimates, respectively, with $95 \%$ level of confidence. ${ }^{36}$

\section{Demographics}

\section{Respondents' characteristics}

Respondent characteristics are provided in table 1 . The majority of pharmacist respondents were managing/ employee pharmacists $76.3 \%$ (491/644), aged 25 to 39 years $59.8 \%(385 / 644)$ and indicated they worked a median 40 hours per week (IQR 37-45 hours); while the majority of pharmacy assistants were dispensary/ general pharmacy assistants $87.9 \%$ (246/280), were 39 years or younger $56.1 \%(157 / 280)$ and indicated they worked a median 34 hours per week (IQR 23-38 hours).

\section{Pharmacy characteristics}

Of the 803 different pharmacies (representing approximately $14.6 \%, 803 / 5500$ of community pharmacies nationally), most were located in areas of high socioeconomic status $(46.6 \%$; $374 / 803)$ followed by low $(31.5 \%$; $253 / 803)$ then medium $(21.9 \%$; 176/803) based on the Socio-Economic Indexes for Areas classification for advantage and disadvantage. ${ }^{37}$ The majority of the pharmacies were classified as highly accessible/metropolitan $(81.8 \%$; $657 / 803)$ followed by accessible/moderately accessible or rural $(12.8 \% ; 103 / 803)$ and remote/very remote $(5.4 \%$; 43/803) according to the Pharmacy Access/Remoteness Index of Australia and the rural pharmacy maintenance allowance agreement. 3839 
Table 1 Characteristics of the pharmacists and pharmacy assistants who responded to the survey

\begin{tabular}{llll}
\hline $\begin{array}{l}\text { Respondent } \\
\text { characteristics }\end{array}$ & Pharmacists (n=644) & Respondent characteristics & Pharmacy \\
\hline $\begin{array}{l}\text { State/Territory } \\
\text { Queensland }\end{array}$ & $25.0 \%(161)$ & Queensland & $23.9 \%(67)$ \\
Northern Territory & $0.9 \%(6)$ & Northern Territory & $0.7 \%(2)$ \\
Australian Capital & $1.1 \%(7)$ & Australian Capital Territory & $1.4 \%(4)$ \\
Territory & & & $27.9 \%(78)$ \\
New South Wales & $31.1 \%(200)$ & New South Wales & $22.9 \%(64)$ \\
Victoria & $19.9 \%(128)$ & Victoria & $3.9 \%(11)$ \\
\hline Tasmania & $2.5 \%(16)$ & Tasmania & $13.6 \%(38)$ \\
Western Australia & $11.8 \%(76)$ & Western Australia & $5.7 \%(16)$ \\
South Australia & $7.8 \%(50)$ & South Australia &
\end{tabular}

Location of pharmacy

based on PhARIA index*

$\begin{array}{llll}\text { Highly accessible } & 80.9 \%(521) & \text { Highly accessible } & 82.1 \%(230) \\ \begin{array}{l}\text { Accessible/moderately } \\ \text { accessible (rural) }\end{array} & 12.9 \%(83) & \text { Accessible/Moderately accessible (rural) } & 12.8 \%(36) \\ \text { Remote/very remote } & 6.2 \%(40) & \text { Remote/very remote } & 5.0 \%(14)\end{array}$

Location of pharmacy

based on SEIFA index

\begin{tabular}{|c|c|c|c|}
\hline High (8-10) & $31.4 \%(202)$ & High (8-10) & $23.9 \%(67)$ \\
\hline Medium (4-7) & $19.3 \%(124)$ & Medium (4-7) & $20.4 \%(57)$ \\
\hline Low (1-3) & $40.7 \%(262)$ & Low (1-3) & $46.8 \%(131)$ \\
\hline \multicolumn{4}{|l|}{ Pharmacy type } \\
\hline Privately owned & $50.8 \%(327)$ & Privately owned & $54.3 \%(152)$ \\
\hline \multicolumn{4}{|l|}{ Pharmacy located in } \\
\hline Shopping strip & $59.6 \%(384)$ & Shopping strip & $53.9 \%(151)$ \\
\hline Shopping centre & $24.5 \%(158)$ & Shopping centre & $29.3 \%(82)$ \\
\hline Health centre & $10.2 \%(66)$ & Health centre & $11.4 \%(32)$ \\
\hline Stand-lone & $1.9 \%(12)$ & Stand-alone & $2.9 \%(8)$ \\
\hline Pharmacy manager & $31.1 \%(200)$ & Dispensary technician & $22.5 \%(63)$ \\
\hline Employee pharmacist & $45.2 \%(291)$ & Retail manager/staff & $6.4 \%(18)$ \\
\hline \multirow[t]{3}{*}{ Intern pharmacist } & $2.2 \%(14)$ & Pharmacy student & $4.3 \%(12)$ \\
\hline & & Naturopath & $0.4 \%(1)$ \\
\hline & & Not completed $\dagger$ & $1.1 \%(3)$ \\
\hline \multicolumn{4}{|l|}{ Age } \\
\hline Under 25 & $9.5 \%(61)$ & Under 25 & $27.9 \%(78)$ \\
\hline $25-29$ & $27.6 \%(178)$ & $25-29$ & $12.1 \%(34)$ \\
\hline
\end{tabular}


Table 1 Continued

\section{Respondent} characteristics

Postgraduate

qualifications

\begin{tabular}{|c|c|c|c|}
\hline Yesł & $20.0 \%$ (129) & Yes & $8.2 \%(23)$ \\
\hline \multicolumn{4}{|c|}{$\begin{array}{l}\text { Prior training/CPD in oral } \\
\text { healthcare }\end{array}$} \\
\hline Yes§ & $16.5 \%(106)$ & Yes & $22.5 \%(63)$ \\
\hline \multicolumn{4}{|c|}{$\begin{array}{l}\text { *PhARIA index } 2 \text { (accessible A), } 3 \text { (accessible B) and } 4 \text { (moderately accessible) have been collapsed into one variable 'accessible/moderately } \\
\text { accessible (rural). PhARIA categories } 5 \text { (remote) and } 6 \text { (very remote) have been collapsed into 'remote/very remote'. } \\
\text { †Indicates respondents did not answer the question. } \\
\text { łTop two postgraduate qualifications reported by pharmacists include Master in Pharmacy and Graduate Diploma in Pharmacy Practice/ } \\
\text { Hospital Pharmacy/Clinical Pharmacy. Top two postgraduate qualifications reported by assistants include Bachelor of Science and Certificate } \\
\text { III Community Pharmacy. } \\
\text { §Top two prior training/CPD in oral healthcare reported by pharmacists include PSA resources/CPD and general or unspecified journal } \\
\text { articles. Pharmacy assistants reported certificates I, II and III training and non-specific online modules from product suppliers for example, } \\
\text { Colgate, and so on. } \\
\text { CPD, continuing personal development; PhARIA, Pharmacy Access/Remoteness Index of Australia; PSA, Pharmaceutical Society of Australia; } \\
\text { SEIFA, Socio-Economic Indexes for Areas. }\end{array}$} \\
\hline
\end{tabular}

Section 1: Oral healthcare service provision and range of OTC products stocked in Australian community pharmacies

In the past 3 months, the majority of pharmacists $(80.2 \% ; 516 / 644)$ and pharmacy assistants $(83.6 \%$; 234/280) reported providing oral health advice/ consultations to health consumers up to five times each week. Approximately one in five pharmacists $(17.2 \%$; $111 / 644)$ and one in 10 assistants (9.3\%; 26/280) provided oral advice/consultations more than six times per week, while only $2.6 \%(17 / 644)$ of pharmacists and $7.1 \%(20 / 280)$ of assistants never provided oral health advice/consultations to consumers. The range of oral healthcare services provided by pharmacists and pharmacy assistants are listed in table 2. Pharmacists and pharmacy assistants reported consulting a range of health consumer groups with the majority providing advice/consultations for adults (pharmacists $93.8 \%$; $604 / 644$ vs $87.1 \%$; $244 / 280$ assistants) and older adults (pharmacists $66.1 \%$; 426/644 vs 56.8\%; $159 / 280$ assistants (table 2). Across Australia, only $15.8 \%(102 / 644)$ of pharmacists and $19.6 \%(55 / 280)$ of assistants referred to guidelines/resources when providing oral health advice/OTC products, with the Therapeutic Guidelines and PSA resources the most common choice for pharmacists and the majority of assistants asked for/referred to the pharmacist for oral health advice/guidance. The majority of pharmacists $(85.2 \%$; 549/644) and pharmacy assistants $(75.4 \% ; 211 / 280)$ believed health consumers were receptive to receiving oral health advice. Cross-tabulation analyses for provision of oral health services and respondent demographic variables showed that pharmacy staff working in privately owned pharmacies $(28.2 \%$; $135 / 479)$ were significantly more likely to follow-up patients regarding previous oral health enquiries compared with buying group pharmacies
$(22.2 \% ; 99 / 445), \mathrm{p}=0.041$. No other relevant associations were identified.

In the past 3 months, approximately $75 \%$ of pharmacists $(72.9 \% ; 470 / 644)$ and pharmacy assistants $(78.9 \%$; $221 / 280)$ recommended up to five OTC oral health products to health consumers each week, with $25.6 \%(165 / 664)$ of pharmacists and $15 \%$ of pharmacy assistants $(42 / 280)$ providing six or more products each week. The proportion of pharmacies stocking various oral healthcare products are listed in online supplementary 2. Cross-tabulation analyses identified significantly fewer pharmacies located in remote/very remote regions compared with pharmacies located in more accessible areas that stocked high-fluoride containing products $(83.7 \% ; 36 / 43$ vs $96 \% ; 631 / 657$, respectively, $\mathrm{p}<0.001)$, chlorhexidine-based mouth products $(81.4 \%$; $35 / 43$ vs $93.2 \%$; $612 / 657$, respectively, $\mathrm{p}=0.022$ ) and fluoride-based mouth rinses $(55.8 \%$; $24 / 43$ vs $71.8 \% ; 472 / 657$, respectively $(\mathrm{p}=0.022)$. Overall, community pharmacies belonging to a buying group were statistically more likely to stock a larger range of oral healthcare products than privately owned pharmacies. No other relevant associations were found.

\section{Section 2: Pharmacy staff attitudes and perceived barriers to providing oral healthcare services and early intervention/ preventive services}

Nearly all pharmacists and the majority of pharmacy assistants strongly agreed or agreed they had an important role to play in promoting oral healthcare in the community (pharmacists $94.1 \%$; 606/644 vs $86.8 \%$; $243 / 280$ assistants), were interested in working collaboratively with other healthcare professionals in their local area (91\%; $586 / 644$ vs $73.9 \% ; 207 / 280$ ), believed that offering oral health programme/services in pharmacy is a good use 
Table 2 Oral healthcare services and health consumer consultations provided by community pharmacists compared with pharmacy assistants

\begin{tabular}{|c|c|c|c|}
\hline & Pharmacists $(n=644)$ & Pharmacy assistants $(n=280)$ & p Value \\
\hline \multicolumn{4}{|l|}{ Oral healthcare services in the past 3 months } \\
\hline Provide OTC treatments for oral health presentations & $94.6 \%(609)$ & $90.7 \%(254)$ & 0.032 \\
\hline $\begin{array}{l}\text { Refer consumers to dental or medical practitioners when } \\
\text { appropriate }\end{array}$ & $93.0 \%(599)$ & $78.2 \%(219)$ & $<0.001$ \\
\hline $\begin{array}{l}\text { Identify signs and symptoms of oral health problems in } \\
\text { patients }\end{array}$ & $72.0 \%(464)$ & $53.9 \%(151)$ & $<0.001$ \\
\hline $\begin{array}{l}\text { Provide guidance and counselling for treatment and } \\
\text { prevention of oral health issues }\end{array}$ & $54.3 \%(350)$ & $39.6 \%(111)$ & $<0.001$ \\
\hline $\begin{array}{l}\text { Opportunistically discuss oral healthcare with patients } \\
\text { where appropriate }\end{array}$ & $47.8 \%(308)$ & $39.3 \%(110)$ & 0.018 \\
\hline Provide oral healthcare related information for patients & $39.9 \%(257)$ & $36.1 \%(101)$ & 0.304 \\
\hline $\begin{array}{l}\text { Follow-up with patients regarding previous oral healthcare } \\
\text { enquiries/consultations }\end{array}$ & $28.7 \%(185)$ & $17.5 \%(49)$ & $<0.001$ \\
\hline Brief clinical examination of patient's oral cavity & $22.2 \%(143)$ & $7.9 \%(22)$ & $<0.001$ \\
\hline $\begin{array}{l}\text { Hold oral health promotion activities to provide education } \\
\text { and raise awareness of issues relevant to oral health }\end{array}$ & $3.1 \%(20)$ & $6.8 \%(19)$ & 0.013 \\
\hline \multicolumn{4}{|l|}{$\begin{array}{l}\text { Pharmacy staff oral health advice/consultation provided for } \\
\text { different consumer groups in the past } 3 \text { months }\end{array}$} \\
\hline Infants ( $0-1$ year) & $36.2 \%(233)$ & $26.8 \%(75)$ & 0.006 \\
\hline Toddler (1-3years) & $37.0 \%(238)$ & $27.5 \%(77)$ & 0.006 \\
\hline Children (4-10years) & $30.6 \%(197)$ & $20.4 \%(57)$ & 0.002 \\
\hline Adolescent (11-19years) & $35.7 \%(230)$ & $27.5 \%(77)$ & 0.015 \\
\hline Adults & $93.8 \%(604)$ & $87.1 \%(244)$ & 0.001 \\
\hline Older adults (>65 years) & $66.1 \%(426)$ & $56.8 \%(159)$ & 0.007 \\
\hline Pregnant/nursing mothers & $17.9 \%(115)$ & $8.9 \%(25)$ & 0.001 \\
\hline
\end{tabular}

OTC, over-the-counter.

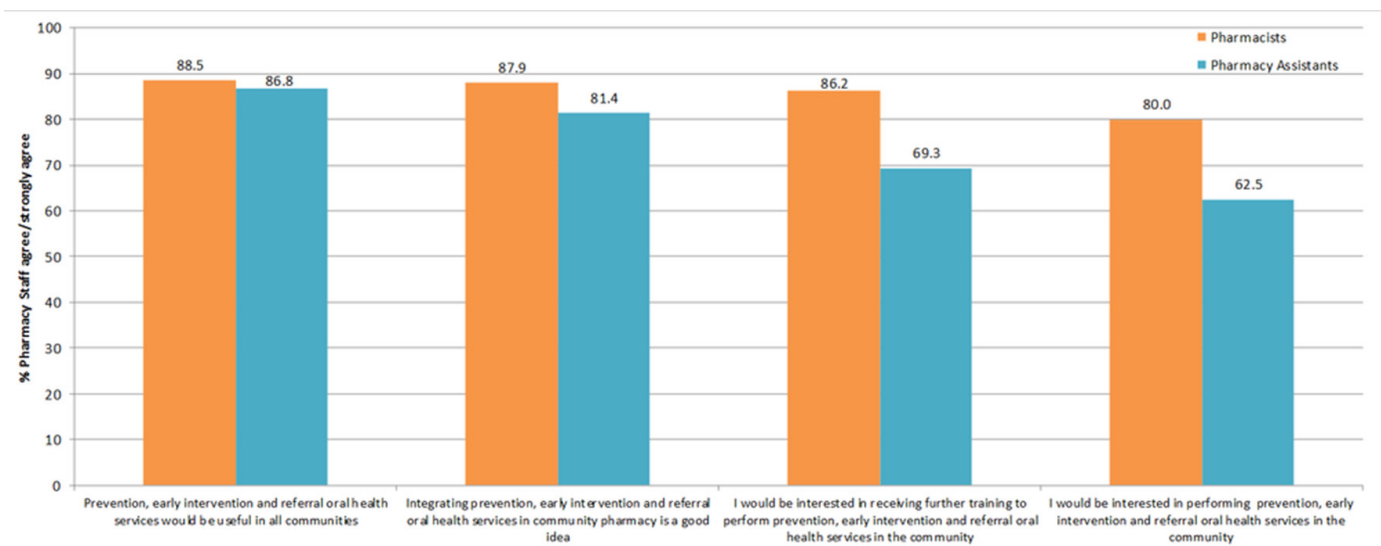

Figure 1 Pharmacy staff (pharmacists $n=644$, pharmacy assistants $n=280$ ) attitudes towards the provision of early intervention, prevention and referral oral health services.

of time/resources $(90.4 \% ; 582 / 644$ vs $82.1 \% ; 230 / 280)$, and were interested in receiving further training to better identify/recognise oral health issues and provide appropriate advice/product recommendations for their clients $(93.5 \% ; 602 / 644 ; 81.4 \% ; 228 / 280)$. The majority of pharmacists strongly agreed or agreed that identifying/ recognising oral health issues and making evidence-based product recommendations was part of their professional responsibility $(84.8 \%$; 546/644), while half of pharmacy assistants $(53.9 \%$; 151) believed this was part of their professional responsibility. Sixty per cent of pharmacists $(386 / 644)$ strongly agreed/agreed being comfortable examining inside a patients' mouth to identify/recognise oral health issues compared with 39.6\% (111/280) 


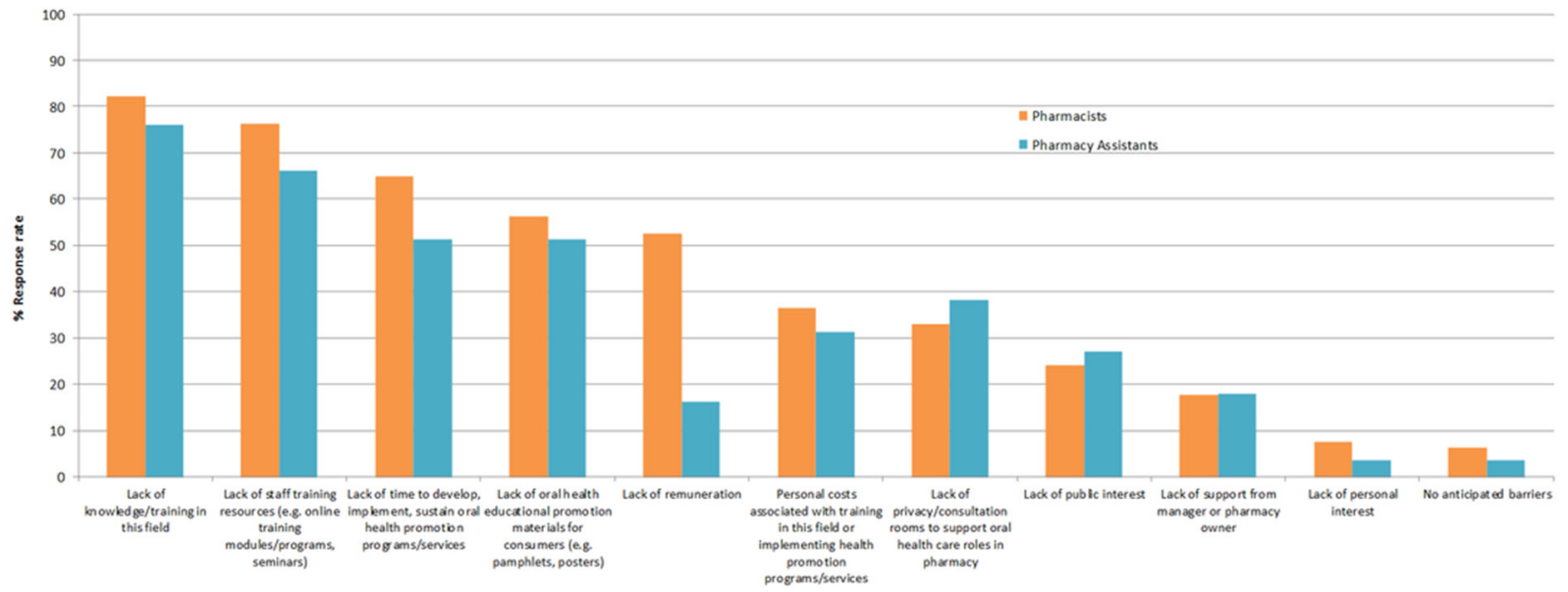

Figure 2 Pharmacy staff (pharmacists $n=644$, pharmacy assistants $n=280$ ) reported barriers to furthering their role in oral healthcare.

of pharmacy assistants. A cross-tabulation analyses of attitudinal-based questions and staff demographic variables showed that pharmacy staff working in remote/very remote pharmacies were significantly more interested in working collaboratively with other healthcare professionals in their local area $(98.1 \% ; 53 / 54)$ compared with staff working in highly accessible/metropolitan $(84.6 \%$; $635 / 751)$ pharmacies $(\mathrm{p}=0.006)$. No other relevant associations were found.

Figure 1 represents community pharmacy staff interest regarding incorporating prevention, early intervention and referral to oral healthcare services within community pharmacies. This includes but is not limited to performing oral health services such as: dental cavity risk assessments (determining the likelihood of caries and their progression in patients); screening and medical/dental practitioner referral programme (eg, 'Lift the Lip programme'-screening patient mouths to detect the presence of cavities and appropriately referring); fluoride varnish application (applying fluoride varnish on patients teeth) and patient education/ guidance to promote and maintain good oral health. ${ }^{10}$ Overall, the majority of pharmacists and assistants viewed these services positively and supported integrating these services into community pharmacy. Pharmacists were also more interested in receiving further training and performing these services than pharmacy assistants. More early career pharmacists (1-5 years after graduation) compared with experienced pharmacists $(20+$ years $)$ were interested in receiving further training $(90.2 \%$; $212 / 235$ vs $78.5 \% ; 117 / 149$, respectively, $\mathrm{p}=0.004)$ and performing prevention, early intervention and referral oral health services $(86 \% ; 202 / 235$ vs $70.5 \% ; 105 / 149$, respectively, $\mathrm{p}=0.002$ ).

Reported barriers preventing pharmacy staff from furthering their role in oral healthcare are given in figure 2. The top three barriers include lack of knowledge and training in the field of oral healthcare, lack of staff training resources and lack of time to develop, implement and sustain oral health promotion programme/services.

\section{Section 3: Training and support required in oral healthcare}

Pharmacists and assistants were asked to rate their confidence in identifying/recognising various oral health conditions. Respondents reporting strongly agree/agree are shown in figure 3. Disparities between pharmacist and pharmacy assistant responses were particularly noted for identifying drug-related adverse oral events (pharmacists $87.1 \%$; $561 / 644$ vs $43.2 \%$; $121 / 280$ assistants), periodontitis (pharmacists $52.2 \%$; 336/644 vs $27.1 \%$; 76/280 assistants) and trigeminal neuralgia (pharmacists $33.5 \%$; $216 / 644$ vs $11.8 \% 33 / 280$ assistants). Less than half of pharmacy staff respondents were confident in recognising 13 out of the 25 oral health conditions.

Factors pharmacy staff feel would improve the ability of Australian pharmacies to better promote oral healthcare are provided in online supplementary 3. Pharmacists were significantly more likely compared with pharmacy assistants to want payment for identifying and managing oral health conditions (69.7\%; 449/644 vs $46.4 \%$; $130 / 280, \mathrm{p}=<0.001)$ and for providing early intervention/ preventive oral health services $(71.1 \%$; $458 / 644$ vs $48.6 \%$; $136 / 280, p=<0.001)$. Preferred distribution methods for educational resources are also provided in online supplementary 3. Pharmacists and pharmacy assistants were also asked their preference for when oral health knowledge/ training would be best delivered. Pharmacists preferred during continuing professional development (CPD) followed by within their undergraduate degree $(87.3 \%$; $562 / 644 \%$ and $60.2 \%$; 388/644 respectively), while pharmacy assistants preferred during their training/studies and CPD equally $(61.8 \% ; 173 / 280$ vs $58.2 \% ; 163 / 280$, respectively).

\section{DISCUSSION}

This study investigated oral health services provided by community pharmacies across Australia. It is the first national study to explore and contrast differences in practice, attitudes and training requirements between pharmacists and pharmacy assistants regarding oral 


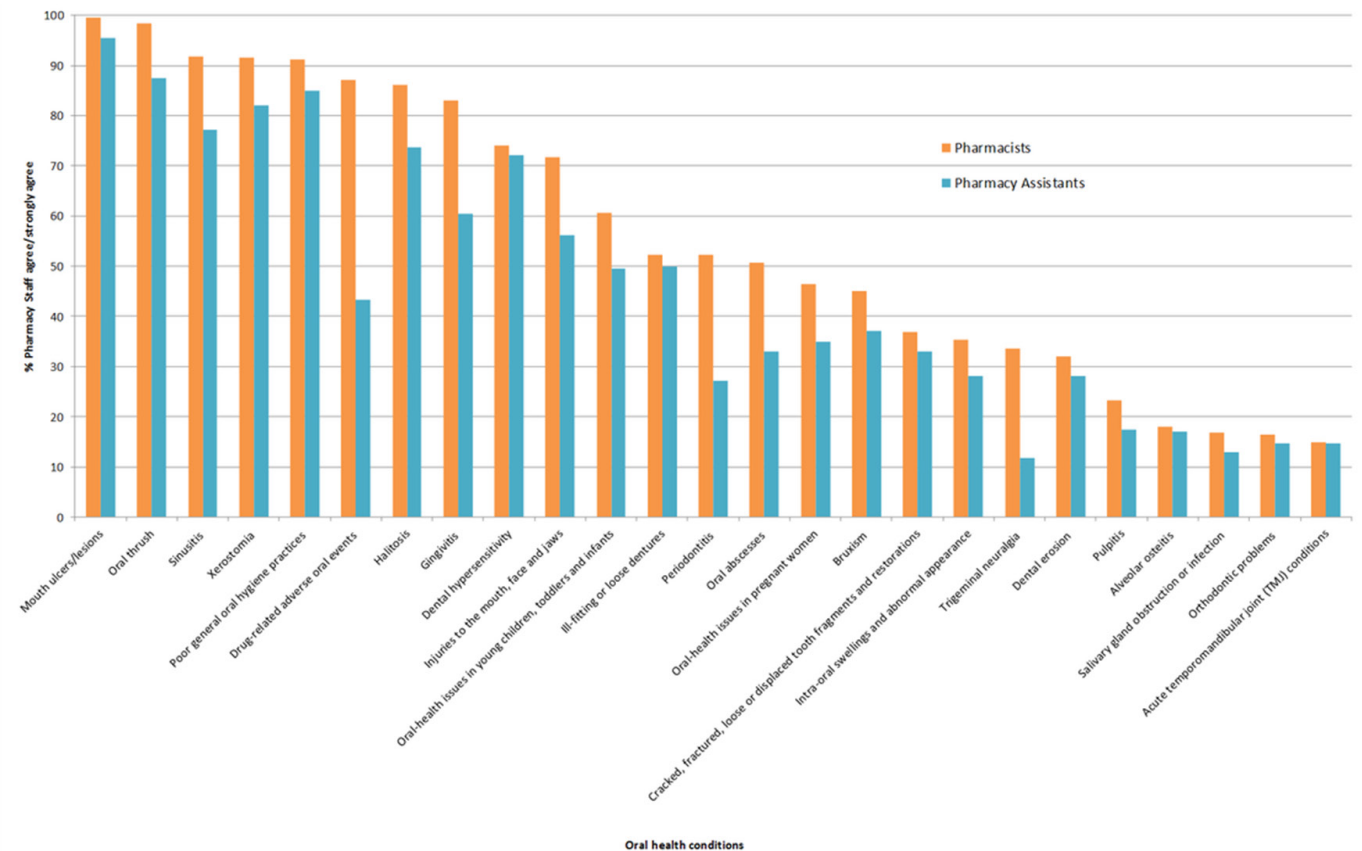

Figure 3 Proportion of pharmacy staff (pharmacists $n=644$, pharmacy assistants $n=280$ ) reporting confidence in identifying/ recognising oral health conditions.

healthcare service provision and is the first to evaluate community pharmacy staff views regarding implementation of prevention, early intervention and referral services which has not been previously assessed.

The findings indicate that more than half of pharmacist and pharmacy assistant respondents were involved in identifying signs and symptoms of oral health problems; and the majority believed health consumers were receptive to receiving oral health advice from them. Australian pharmacists play a valuable role in primary healthcare by managing or triaging minor ailments in the community and continue to be consulted as they are an accessible and trusted source for health advice. ${ }^{40}{ }^{41}$ Interestingly, half of the pharmacy assistant respondents in this study reported being involved in identifying signs/symptoms for oral health conditions in patients. Because pharmacists and pharmacy assistants are involved in recognising/ identifying oral health conditions in the community, it is imperative that they are trained with the appropriate knowledge and skills to differentially identify/refer common oral health presentations when necessary. Our previous research showed the three most commonly identified oral presentations in community pharmacies include mouth ulcers, oral thrush and toothache. ${ }^{29}$ It is not clear why patients present community pharmacy staff with oral health issues, however, barriers to accessing dental care comprising psychosocial factors such as dental anxiety, financial costs, perceptions of need and lack of access are likely reasons. ${ }^{42}$ It is not known whether Australian pharmacy staff are appropriately identifying and managing oral health presentations within the community and future studies using methodologies including standardised patient (mystery shopper study) and/or case vignettes will be necessary to assess this.
This study showed only half or less of all pharmacy staff provide guidance and counselling for treatment and prevention of oral health issues. This may be due to several reasons including lack of public interest, consumer self-selection of oral health products away from staffed counters/services areas, lack of private counselling areas and potentially time, however, two predominant and consistent themes reported by staff preventing them from participating in treatment/prevention of oral health issues was their lack of knowledge/ongoing training in the field and resources to assist practice (unpublished qualitative data). These two factors were also the most commonly reported barriers (or conversely, facilitators necessary) to furthering pharmacy staff roles in oral healthcare. This finding is not surprising given the lack of pharmacy-specific practice guidelines, decision support pathways, information and educational resources to assist staff during oral health promotion/management of oral health issues. An evaluation (by authors MWT and $\mathrm{CF}$ ) of Australian pharmacy resources (online and printed) to support practice in oral healthcare revealed only one published pharmacy-specific practice guideline to manage mouth ulcer presentations. ${ }^{43}$ Currently, there are no published studies describing the level or scope of education provided to pharmacists during undergraduate/postgraduate studies or within training programme offered to pharmacy assistants. Anecdotal reports via pharmacy academics within Queensland and New South Wales, however, reveal pharmacy students are provided on average only two lectures throughout their entire programme relating to oral health and healthcare in the community. Given the minimal level of oral health education and pharmacy-related system level barriers (eg, time constraints) experienced by pharmacy staff, a practical 
first step could involve interdisciplinary partnerships to develop pharmacy-specific evidence-based guidance (eg, clinical decision pathways) on oral healthcare topics identified as priorities for oral healthcare in Australia. This is in concert with the national goals outlined in Australia's National Oral Health Plan 2015-2024 which includes providing all Australians access to oral health promoting environments and appropriate evidence-based information and programmes that support them to make informed decisions about their oral health. ${ }^{44}$ Pharmacy staff would then be better equipped to intervene/provide oral health advice to consumers when purchasing oral health-related products within community pharmacies. Additionally, oral health products may be positioned closer to service counters to promote oral health promotion activities and particular products may be flagged in point of sale or dispensary systems to enhance advice provision and oral healthcare (eg, those used to manage adverse drug effects such as xerostomia).

This study showed that only half or less of pharmacists and pharmacy assistants viewed lack of remuneration for services as a barrier for furthering their role in oral healthcare (ranked fifth and ninth highest out of 10 for pharmacists and pharmacy assistants, respectively). This finding is in contrast to pharmacy literature identifying lack of remuneration as a key barrier preventing greater provision of clinical services; which is likely due to diminishing dispensary revenues in light of generic drug price reductions/disclosures and other factors. ${ }^{45} 46$ The majority of pharmacists and assistants in this study preferred online training during continuing professional development (CPD) followed by training within their undergraduate degree or studies. Approximately one-third of pharmacists and pharmacy assistants were also open to undertaking a formal course for further training. This presents an opportunity for Australian pharmacy professional bodies to form collaborative partnerships with Australian dental associations/schools to develop evidence-based CPD programmes or formal courses in oral healthcare tailored for community pharmacy staff. This study also highlights the potential to use these collaborations to further expand the scope and extent of oral health education during training for pharmacy assistants and within pharmacy student undergraduate and postgraduate teaching curricula so that the profession is better equipped to handle consumer oral health enquiries in practice.

In the majority of cases, high costs associated with dental treatment can be avoided by effective prevention and health promotion measures. ${ }^{1510}$ This study is the first to assess Australian pharmacy staff attitudes with regards to potential engagement in prevention, early intervention and referral to oral healthcare services within community pharmacies. The majority of pharmacists and pharmacy assistants viewed these services positively and supported integrating them within their workplace. Younger, early career pharmacists and pharmacists as a professional group compared with pharmacy assistants were more inclined to adopt these new initiatives as part of their oral healthcare role in the community. Despite a reduction of reported dental caries in children and tooth loss in adults over the past 30 years, oral diseases and disorders in Australia remain prevalent and are a substantial burden on the population. ${ }^{47}$ There is need for a continuing and robust service system supporting access across communities for prevention, treatment and specialist services for specific groups with particular needs. ${ }^{48}$ Disadvantaged groups in particular (eg, low-income earners, indigenous, the elderly and remote/rural populations) are at higher risk of poor oral health and are less likely to receive preventive oral care services. The potential for community pharmacies to integrate prevention, early intervention and referral services/programmes is an attractive proposition that may be highly beneficial particularly in disadvantaged populations due to geographical or socioeconomic inequalities in access. For example, people living in Australian regional and remote areas experience poorer oral health at significantly higher rates than the general population. ${ }^{49}$ A cross-tabulation analysis of pharmacy staff demographics showed that staff working in rural or remote communities compared with highly accessible (metropolitan) regions were less likely to report lack of manager/pharmacy owner support as a barrier towards furthering their role in oral healthcare and were also more interested in working collaboratively with other healthcare professionals in their local area to support better oral health. A major advantage of engaging community pharmacists over other health professions to perform prevention, early intervention and referral oral health programmes/services stems from their partnership with consumers over a life course in chronic disease management combined with ease of population access including for those without pre-existing conditions. Given the oral health inequalities experienced in Australian rural/remote communities, this study identifies the potential for rural/remote community pharmacy practices to form interprofessional healthcare partnerships with dental professionals to implement preventive oral care services in their region to play a larger role in improving the oral health needs in these communities. However, it is necessary to engage and partner with relevant stakeholders to direct the development of educational programme/resources tailored to pharmacy staff and to inform design of innovative evidence-based intervention/practice models that will require piloting to assess implementation considerations such as feasibility, acceptability and importantly cost-benefit modelling/ analyses.

The Institute of Medicine stipulates interprofessional collaboration as a core competency that all health professionals should strive to achieve during provision of patient care. ${ }^{50}$ Pharmacists and dental practitioners have responsibilities for improved patient health outcomes and share complementary roles including diagnosis or recognition of disease and disorders, preventing disease, promoting better health, treatment and monitoring diseases/ 
disorders and ensuring quality use of medicines. ${ }^{51}$ These shared and complementary responsibilities provide an excellent opportunity for collaboration with regard to sharing and reviewing medical and medication histories, pain management, prevention and management of infections and referral of patients. ${ }^{51}$ For example, if a dental practitioner perceives that a certain medication may contribute to increased risk of perioperative complications, through sharing and reviewing of medical and medication histories, pharmacists can assist dentists in determining which medicines may contribute to risk for example, bisphosphonates, denosumab, nonsteroidal anti-inflammatory drugs and herbal medicines. There are many common adverse drug effects that manifest in the oral cavity (eg, xerostomia, tooth discolouration and immunosuppression/increased risk of infection) that through pharmacist consultation/collaboration, could be identified, managed or prevented. Dentists may also collaborate with pharmacists to inform specific oral care products commonly recommended and refer patients to pharmacists for vaccinations, gloves, face masks, smoking cessation products and general healthcare advice. Depending on jurisdiction regulations, guidelines could be developed collaboratively where dentists refer patients to pharmacists for naloxone, self-administered hormonal contraception, travel medicine and ordering of laboratory studies to monitor diseases. It is also important to consider medical prescribers, who see many patients with oral and dental problems and may collaborate and work with pharmacists and dentists in new ways to improve patient health outcomes. Importantly, for any service or model to work effectively, there must be a desire for professional stakeholders to want to work together, build mutual respect and trust for one another and understand the roles of each profession with regard to improved patient care.

A limitation of this study is that findings were self-reported and may be subject to respondent recall and social desirability bias. In this study, the pharmacist and pharmacy assistant survey had a low response rate $(58.5 \%$ and $28 \%$, respectively); however, this is comparable to or relatively higher than many Australian and International pharmacy-based surveys which report response rates of approximately $18 \%-25 \%$. There are a number of reasons that could contribute towards low response rates, the likely and most commonly reported within this sector is that pharmacy staff are a time poor and oversurveyed population group in Australia. ${ }^{29}$ The sample size obtained in this study allowed us to capture a range of Australian pharmacist and pharmacy assistant responses, however, as non-respondent characteristics were unable to be examined (due to non-participation and therefore lack of important demographic and attitudinal variables and reasons for non-response), this may potentially limit the generalisability of the findings. Our previous study ${ }^{29}$ showed that both pharmacists and pharmacy assistants were involved in handling the majority of oral health-related presentations in Australian community pharmacies, hence a valuable aspect of this study was its design to obtain responses from both pharmacist and pharmacy assistants. Furthermore, the questionnaire was piloted for content and face validity by a multidisciplinary research team and practising pharmacists and pharmacy assistants.

In Australia, pharmacy and dental professional groups recognise community pharmacists can collaboratively play a pivotal role in improving the oral health of consumers. Collaborative work has evolved from the launch of the ADA Victoria Branch, Dental Health Services Victoria and Pharmacy Society of Australia Victoria Branch via development of a joint position statement on oral health and pharmacy in $2015 .^{52}$ This work has been extended through formation of the 'Pharmacy and Oral Health Alliance' in 2016 (a group of policy-makers, dental practitioners and pharmacists from several tertiary institutions), who aim to promote better oral health through the pharmacy sector at a national level. In Australia, the traditional role of pharmacy as supplier of medicines has been challenged given reduction of pharmaceutical benefits scheme dispensing margins. This has encouraged the pharmacy industry to implement new and sustainable services which best use pharmacy staff knowledge and expertise. Pharmacies are readily accessible to consumers, consist of a workforce of highly qualified health professionals with a strong professional commitment to integrated healthcare and are regularly consulted by consumers for oral healthcare advice. These factors highlight the potential to extend the current use of pharmacy infrastructure to enhance the role of pharmacy staff in oral healthcare and accommodate the provision of professional preventive oral healthcare services. This will inevitably promote further interprofessional collaborations to support the dental workforce. In conclusion, this study provides evidence supporting the need for growing partnerships/ collaborations between pharmacy and dental healthcare professionals and organisations to develop, implement and evaluate evidence-based resources, interventions and services to deliver improved and responsive oral healthcare within all Australian communities.

Contributors MWT, PF, CF developed the survey questionnaires. MWT and NA completed the analysis of the survey data. MWT drafted the manuscript. All authors conceived the study and participated in its design and read and approved the final manuscript. All Authors state that they had complete access to the study data that support the publication.

Funding This work was kindly supported by an Alliance for a Cavity Free Future community grant funded by Colgate. We would like to acknowledge the work of UQ pharmacy students Lucas Panagopoulos, Cathryn Carlisle and Chi Ying Tan, with assisting in the development of the Australian community pharmacy premises list, recruiting participants and collecting survey data for this study.

Competing interests None declared.

Ethics approval The University of Queensland's Human Research Ethics Committee.

Provenance and peer review Not commissioned; externally peer reviewed. Data sharing statement No additional data are available.

Open Access This is an Open Access article distributed in accordance with the Creative Commons Attribution Non Commercial (CC BY-NC 4.0) license, which permits others to distribute, remix, adapt, build upon this work non-commercially, 
and license their derivative works on different terms, provided the original work is properly cited and the use is non-commercial. See: http://creativecommons.org/ licenses/by-nc/4.0/

(C) Article author(s) (or their employer(s) unless otherwise stated in the text of the article) 2017. All rights reserved. No commercial use is permitted unless otherwise expressly granted.

\section{REFERENCES}

1. World Health Organisation. Oral health. 2015 http://www.who.int/ topics/oral_health/en/.

2. AlHW. Health and welfare expenditure series no. 42. 2010. Cat. no. HW51 http://www.aihw.gov.au/publication-detail/?id=6442472450

3. Australian Institute of Health and Welfare. How much does Australia spend on health care. Canberra: AIWH, 2014.

4. Jill Hall SI, Coulton M, Georganas S, et al. In: H.S.C.o.H.a. Ageing, Ed. Inquiry into adult dental services in Australia. Canberra: Printing and Publishing Office, 2013;75

5. Suga US, Terada RS, Ubaldini AL, et al. Factors that drive dentists towards or away from dental caries preventive measures: systematic review and metasummary. PLoS One 2014;9:e107831.

6. Rogers JG. Evidence-based oral health promotion resource. Prevention and Population Health Branch, Department of Health, 2011.

7. Hobdell M, Petersen PE, Clarkson J, et al. Global goals for oral health 2020. Int Dent J 2003;53:285-8.

8. Chalmers JM, King PL, Spencer AJ, et al. The oral health assessment tool-validity and reliability. Aust Dent J 2005;50:191-9.

9. Rozier RG, Sutton BK, Bawden JW, et al. Prevention of early childhood caries in North Carolina medical practices: implications for research and practice. J Dent Educ 2003;67:876-85.

10. Calache $\mathrm{H}$, Hopcraft MS, Martin JM. Minimum intervention dentistry - a new horizon in public oral health care. Aust Dent $J$ 2013;58(Suppl 1):17-25.

11. Arrow $P$, Raheb J, Miller M. Brief oral health promotion intervention among parents of young children to reduce early childhood dental decay. BMC Public Health 2013;13:245.

12. The Pharmacy Guild of Australia. Community Pharmacy, a trusted public-private partnership delivering accessible high quality healthcare for all Australians. 2014 https://www.guild.org.au/docs/ default-source/public-documents/news-and-events/media-releases/ 2014/pgoa-submission-to-the-competition-policy-review-june-2014 573492 3.pdf?sfvrsn=2

13. The Pharmacy Guild of Australia. Community pharmacy: delivering accessibility, quality and choice for all Australians, in Submission in resonse to the competition policy review draft report. 2014

14. Benrimoj SI, Frommer MS. Community pharmacy in Australia. Aust Health Rev 2004;28:238-46.

15. Fakih S, Marriott JL, Boardman $\mathrm{H}$, et al. Comparing women pharmacy consumers' experiences with weight loss treatment in Victoria and Nottingham: a cross-sectional study. BMC Public Health 2014;14:662

16. Joyce AW, Sunderland VB, Burrows S, et al. Community pharmacy's role in promoting healthy behaviours. J Pharm Pract Res 2007;37:42-4.

17. Greenberg BJ, Kumar JV, Stevenson H. Dental case management: increasing access to oral health care for families and children with low incomes. J Am Dent Assoc 2008;139:1114-21.

18. Kiyak HA, Reichmuth M. Barriers to and enablers of older adults' use of dental services. J Dent Educ 2005;69:975-86.

19. Cohen LA. Enhancing pharmacists' role as oral health advisors. J Am Pharm Assoc 2013:53:316-21.

20. Tonetti MS, Van Dyke TE. Working group 1 of the joint EFP/AAP workshop. Periodontitis and atherosclerotic cardiovascular disease: consensus report of the Joint EFP/AAP Workshop on Periodontitis and Systemic Diseases. J Clin Periodontol 2013;40(Suppl 14):S24-9.

21. Ship JA. Diabetes and oral health: an overview. J Am Dent Assoc 2003;134(Suppl1):4S-10.

22. Committee on an Oral Health. I and M Institute of, advancing oral health in America. Washington, DC, USA: National Academies Press, 2011.

23. Australian Dental Association. Policy statement 3.14 -The role of non-dental practitioners in oral health. ADA, 2015;2.
24. PSA. Solutions for an effective 6th Community Pharmacy Agreement (6CPA), 2014. http://www.psa.org.au/download/submissions/6cpasummary-flyer.pdf

25. Angley M, Rigby D, Dowling HV, et al. Advocating for Patients and the Pharmacist's Role in Primary Care. ऐ Pharm Pract Res 2014;44:6-9.

26. George PP, Molina JA, Cheah J, et al. The evolving role of the community pharmacist in chronic disease management - a literature review. Ann Acad Med Singapore 2010;39:861-7.

27. Maunder PE, Landes DP. An evaluation of the role played by community pharmacies in oral healthcare situated in a primary care trust in the north of England. Br Dent J 2005;199:219-23.

28. Gilbert $\mathrm{L}$. The role of the community pharmacist as an oral health adviser-an exploratory study of community pharmacists in Johannesburg, South Africa. SADJ 1998;53:439-43.

29. Taing MW, Ford PJ, Gartner CE, et al. Describing the role of Australian community pharmacists in oral healthcare. Int J Pharm Pract 2016;24:237-46.

30. Priya S, Madan Kumar PD, Ramachandran S. Knowledge and attitudes of pharmacists regarding oral health care and oral hygiene products in Chennai city. Indian J Dent Res 2008;19:104.

31. Jorgenson D, Lamb D, MacKinnon NJ, et al. Practice change challenges and priorities: a National Survey of Practising Pharmacists. Can Pharm J 2011;144:125-31.

32. Steel BJ, Wharton C. Pharmacy counter assistants and oral health promotion: an exploratory study. Br Dent J 2011;211:E19.

33. Mann RS, Marcenes W, Gillam DG. Is there a role for community pharmacists in promoting oral health? Br Dent J 2015;218:E10.

34. Taing MW, Tan ET, Williams GM, et al. Herbal and nutrient complementary medicines for weight loss: community pharmacists' practices, attitudes, recommendations, information and education needs. Int J Pharm Pract 2016;24.

35. IBM Support. Interpreting adjusted residuals in Crosstabs cell statistics. 2016 https://www-304.ibm.com/support/docview.wss? uid=swg21479605

36. Raosoft. Sample size calculator. 2004 http://www.raosoft.com/ samplesize.html

37. ABS. Socio-economic Indexes for Areas (SEIFA), Data Cube only Australia: Australian Bureau of Statistics, 2013.

38. 6th Community Pharmacy Agreement. Rural Pharmacy Maintenance Allowance 2015 http://6cpa.com.au/rural-support-programmes/ruralpharmacy-maintenance-allowance/

39. Hugo Centre for Migration and Population Research. Pharmacy access/remoteness index of Australia 2016 http://www.adelaide.edu. au/apmrc/research/projects/pharia/pharia-info.html

40. Rigby D. Collaboration between doctors and pharmacists in the community. Aust Prescr 2010;33:191-3.

41. Morgan R. Roy Morgan image of professions survey. 2016;2016.

42. Freeman R. The psychology of dental patient care: Barriers to accessing dental care: patient factor. Br Dent J 1999;187:141-4.

43. Sansom LN. Australian pharmaceutical formulary and handbook. 23rd ed. Canberra: Pharmaceutical Society of Australia, 2015.

44. Oral Health Monitoring Group, COAG Health Council, Ed. Australia's National Oral Health Plan 2015-2024. Adelaide: South Australian Dental Service, 2015.

45. Houle SK, Grindrod KA, Chatterley T, et al. Paying pharmacists for patient care: A systematic review of remunerated pharmacy clinical care services. Can Pharm J 2014;147:209-32.

46. Houle SKD, Grindrod KA, Chatterley T, et al. Paying pharmacists for patient care. Can Pharm J 2014;147:209-32.

47. Brennan DS, Balasubramanian M, Spencer AJ. Trends in dental service provision in Australia: 1983-1984 to 2009-2010. Int Dent J 2015;65:39-44.

48. Schwarz E. Access to oral health care - an Australian perspective. Community Dent Oral Epidemiol 2006;34:225-31.

49. Laverty M, Bishop L. Filling the gap disparities in oral health access and outcomes in remote and rural Australia, 2016.

50. Medicine lo. Health professions education: a bridge to quality. Washington, DC, 2003.

51. Valle-Oseguera C, Boyce EG. Dentists and Pharmacists: Paradigm Shifts and Interprofessional Collaborative Practice Models. J Calif Dent Assoc 2015;43:591-5.

52. Pharmaceutical society of Australia. Peak bodies collaborate to improve oral health in Victoria. 2015 http://www.psa.org.au/mediareleases/peak-bodies-collaborate-to-improve-oral-health-in-victoria 